\title{
Low temperature and conventional scanning electron microscopic observations of dog femoral condylar cartilage surface after anterior cruciate ligament division
}

\author{
PATRICIA O'CONNOR, ${ }^{1} \mathrm{~K}$ OATES, ${ }^{2}$ D L GARDNER, ${ }^{1} \mathrm{~J}$ F S MIDDLETON ${ }^{2}$ \\ CONSTANCE R ORFORD, ${ }^{1}$ AND JANET D BRERETON ${ }^{1}$
}

From the ${ }^{1}$ Department of Histopathology, University of Manchester; and the ${ }^{2}$ Department of Biological Sciences, University of Lancaster

SUMmaRY The left stifle joints of 16 beagle dogs were subjected to surgical division of the anterior cruciate ligament (ACL). The femoral condyles showed surface alterations and damage after 14 days when examined by low temperature (LTSEM) and conventional scanning electron microscopy (SEM). The first change was an increase in the fibrous texture of the articular cartilage surface. Folds and surface roughening were later recognised. After 112 days deformation and disruption gave way to cracking or fracture of the surface layers. Superficial damage was focal in distribution and accompanied by a decrease in the frequency of tertiary elevations. The affected areas of cartilage increased in frequency and size with time after surgery. The observations obtained from LTSEM, in which the tissue remains fully hydrated during examination, may be caused by alterations in water content and a loss of proteoglycan at the surface, together with changes in cell shape and size. Surface cracks may be the en face appearance of histological fibrillation.

Key words: frozen hydrated, experimental model osteoarthrosis.

This paper describes the scanning electron microscopic (SEM) and low temperature SEM (LTSEM) appearances of dog femoral condylar cartilage surfaces one to 168 days after surgical division of an anterior cruciate ligament (ACL). Resection of knee joint ligaments, widely practised since its introduction by Magnuson, ${ }^{1}$ has provided a useful and reproducible means of causing prolonged experimental joint disease. ${ }^{2-4}$ Anterior cruciate ligament division results in a brisk sterile synovitis ${ }^{5}$ and mechanical instability. A sequence of histological changes has been recorded, including an initial increase in cell density, a depletion of metachromatic material, and a gradual but conspicuous increase in cartilage thickness (Gardner, personal com-

Accepted for publication 21 November 1984.

Correspondence to Dr P O'Connor, Department of Histopathology, University Hospital of South Manchester, Nell Lane, West Didsbury, Manchester M20 8LR. munication). At the ultrastructural level inappropriate cell morphology in the superficial zone ${ }^{6}$ and a separation of the collagen fibrils ${ }^{67}$ have been observed. These changes in morphology are accompanied by alterations in the chemical composition of the cartilage: increases in water content, dry weight, deoxyribonucleic acid (DNA) and collagen synthesis, and changes in proteoglycan molecular size and aggregation have been described. ${ }^{89}$ The present study was designed to investigate whether these histological and chemical changes are reflected in ultrastructural alterations at the articulating surfaces.

\section{Materials and methods}

Closed surgical division of the ACL was performed on the left stifle joint of 16 normal, inbred beagle dogs aged 9-20 months, weighing $8 \cdot 1-18 \cdot 3 \mathrm{~kg}$. The right knee acted as a sham-operated control in which 
the joint capsule was opened but the ligament left intact. Details of the surgical procedure are given elsewhere. ${ }^{6}$ Twenty animals aged 8-15 months, weighing 7-15 kg were used as normal unoperated controls.

\section{LOW TEMPERATURE SEM}

Cartilage blocks, $2.0 \times 2.0 \times 0.5 \mathrm{~mm}$, were collected from the load-bearing areas of the medial femoral condyles of all operated and sham-operated stifle joints. Similar specimens were obtained from the lateral condyles after they had been washed with $0.9 \%$ sodium chloride. Additional blocks were collected from areas adjacent to macroscopic damage. Comparable blocks were also taken from the medial and lateral femoral condyles of 14 unoperated dogs.

Each cartilage specimen was attached to an aluminium stub with conductive carbon cement (CCC) (Neubauer Chemikalien) and immediately quenched and frozen in nitrogen slush $(63 \mathrm{~K})$. The tissue was stored in liquid nitrogen $(78 \mathrm{~K})$ before examination. Location of the specimen on a cold pedestal $(89 \mathrm{~K})$ in the antechamber (airlock) of a modified Jeol JSM 50A SEM was accomplished with a transfer device. ${ }^{10}$ The specimen was then positioned on a low temperature stage $(81 \mathrm{~K})$ and observed at an accelerating voltage of $10 \mathrm{kV}$ and a beam current of $0.4 \mathrm{nA}$. Surfaces masked by frozen synovial fluid or saline were etched by raising the temperature of the stage to $203 \mathrm{~K}$; this is above the temperature at which sublimation of water occurs (approx. $143 \mathrm{~K}$ ). The stage temperature was then rapidly returned to $81 \mathrm{~K}$ for examination at $10 \mathrm{kV}$. Each specimen was repositioned in the airlock for coating with an approx. $4 \mathrm{~nm}$ layer of carbon followed by approx. $25 \mathrm{~nm}$ of gold by high vacuum evaporation. Further observation continued at 20 $\mathrm{kV}$ and $0 \cdot 1 \mathrm{nA}$.

In order to interpret the LTSEM observations it was first necessary to identify the effects of thermal etching on the structural integrity of the cartilage surfaces. Six frozen cartilage blocks from the femoral condyles of unoperated joints were studied before and after controlled etching. A selected surface area on each block was observed and photographed at $81 \mathrm{~K}$ before the stage temperature was raised to $223 \mathrm{~K}$. The temperature was then rapidly returned to $81 \mathrm{~K}$ for re-examination.

\section{CONVENTIONAL SEM}

Blocks of articular cartilage, $2.0 \times 2.0 \times 0.5 \mathrm{~mm}$, were taken from the main load-bearing regions of the lateral femoral condyles of both left and right stifle joints of the 16 operated dogs after the surfaces had been washed with $0.9 \%$ sodium chloride. Compar- able blocks from 14 unoperated dogs were als르. collected for examination. All specimens were? prepared by the technique described by Cameron Gardner, and Longmore. " Tissue blocks were fixe in $2.5 \%$ glutaraldehyde buffered to $\mathrm{pH} 7.4$ witls sodium cacodylate. After washing, the tissue waş dehydrated with ethanol and vacuum dried frono propylene oxide. Blocks were sputter coated with gold-palladium before examination in a Cambridge S4-10 SEM with a beam current of $0 \cdot 15-0 \cdot 20 \mathrm{nA}$ an $\vec{\Phi}$ an accelerating voltage of $20 \mathrm{kV}$.

\section{Results}

A postmortem examination of the femoral condyla $\vec{f}$ cartilage of seven left stifle joints showed evidence्ध of macroscopic damage. Two animals, one killed $15 \mathrm{~J}$ days and the other 84 days after surgery, each had $\Phi$ single deep cut on the medial condyle. These artefacts were thought to have been accidentally

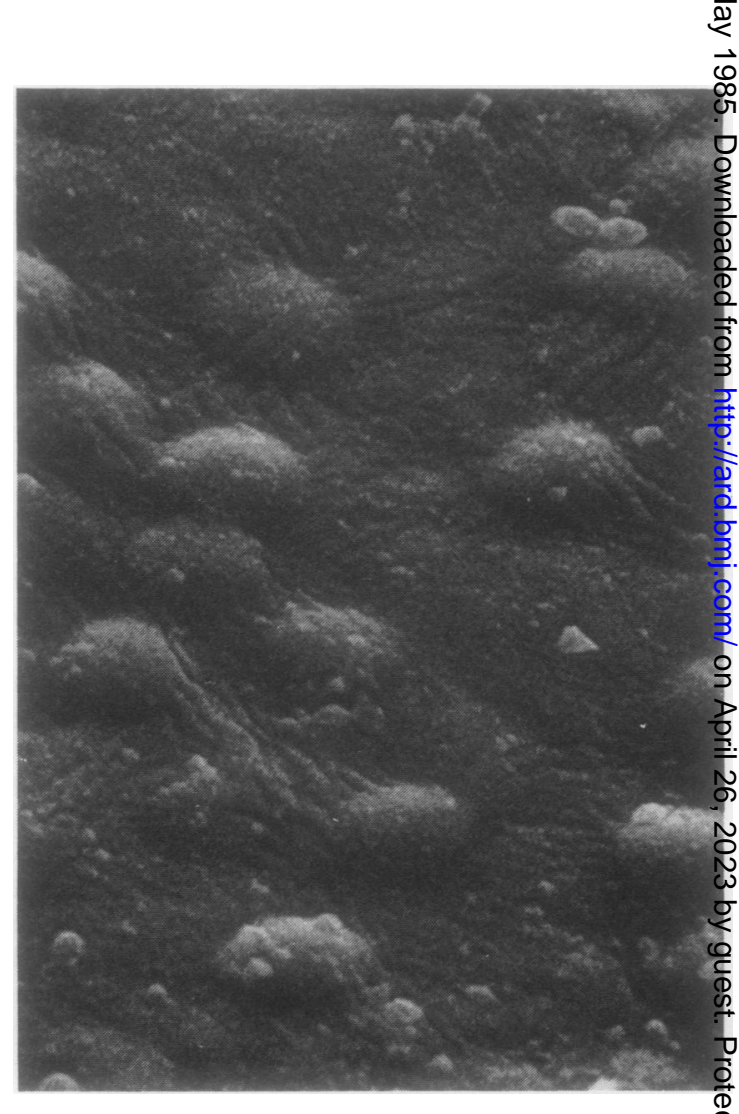

Fig. 1 Frozen hydrated articular cartilage from the mediaf femoral condyle of a normal canine stifle joint. The surfac@ is covered by an array of tertiary elevations and quaternar ridges. LTSEM. $(\times 1110)$. 
introduced during surgery, a disadvantage of the closed operative procedures. Another five dogs killed 28, 56, 112 ( 2 dogs), and 168 days after ACL division possessed areas of gross surface roughening, cartilage thinning, or erosion detectable by the naked eye. In only one dog, killed after 112 days, did this damage occur in the lateral condyle. Osteophytes were present in the left knee joints of dogs killed 112 and 168 days after surgery.

\section{LOW TEMPERATURE SEM}

\section{Normal joints}

The articular surfaces of washed and unwashed blocks from normal control joints have been described previously. ${ }^{10}$ They consisted of a random array of single tertiary elevations of varying diameters and heights (Fig. 1). The elevations were more frequent and of larger diameter on washed than on unwashed surfaces.

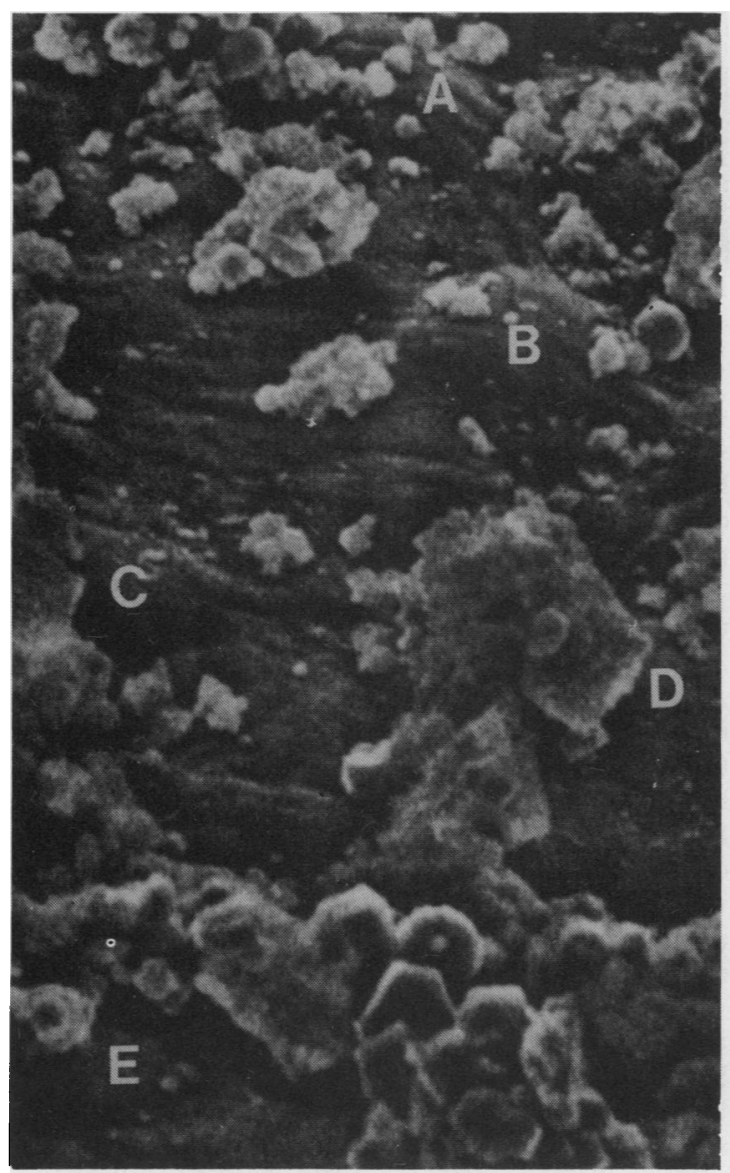

Fig. 2(a) Surface of frozen hydrated normal articular cartilage covered by ice deposits and frozen synovial fluid.
In the areas between elevations, quaternary ridges were frequently observed either radiating from an elevation, forming a criss-cross pattern, or arranged as parallel arrays (Fig. 1). The surface appeared granular at magnifications above 1000 .

Thermal etching removed much of the ice deposit which previously obscured regions of the cartilage surface. Areas not covered by ice showed similar features before and after etching (Fig. 2). The only alteration detected after etching was an increased definition of the quaternary ridges.

\section{Operated joints}

Material collected from one to 14 days after surgery appeared normal.

Fourteen days after surgery fissures developed on the surface and became more widespread with time. In some instances it was difficult to determine

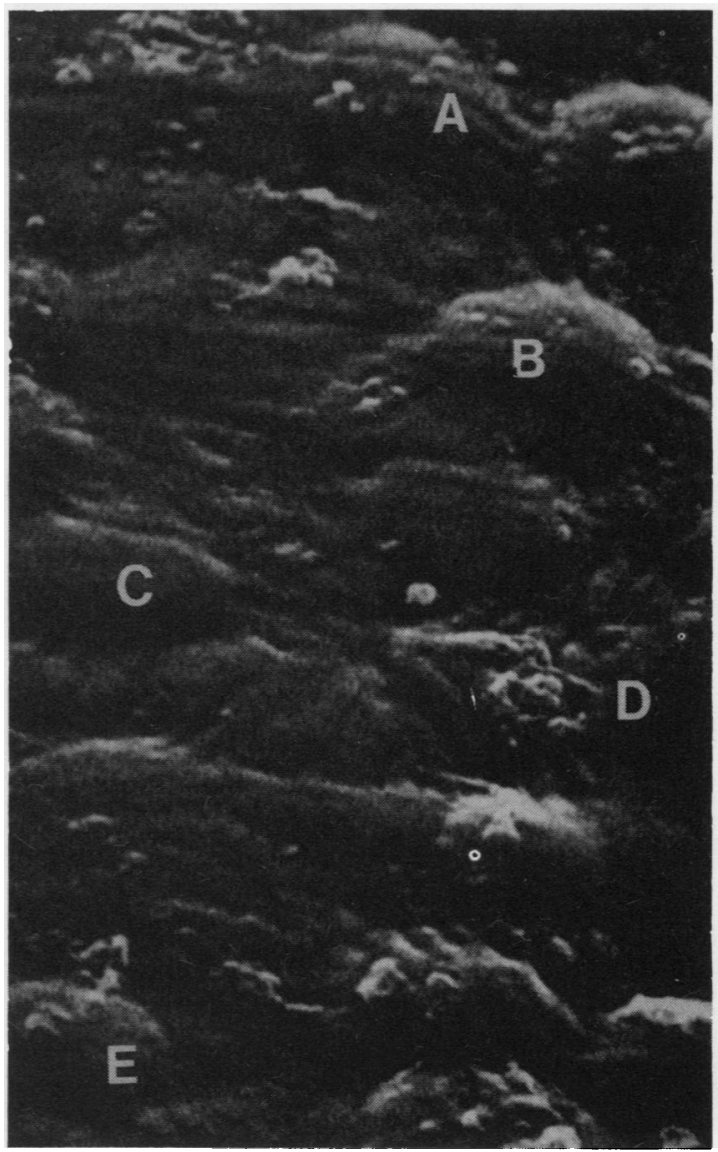

(b) Same surface after raising the stage temperature to $223 \mathrm{~K}$ to remove frozen deposits. Letters mark identical sites in each micrograph. LTSEM. $(\times 1250)$. 
whether fissures represented cracks and thus fractures of the surface or whether they were a form of distortion caused by deformation of the superficial layers. Detailed examination frequently showed narrow, steep-sided troughs with smooth, scalloped walls and no evidence of fracture (Fig. 3); material in the base of the trough occasionally showed a fibrous texture. Between 28 and 56 days after surgery fissuring was localised and took the form of long meandering troughs with secondary folds at right angles to the main direction of distortion. By 84 days the fissures had increased in frequency and were often associated with severe deformation. In areas of gross distortion it was difficult to distinguish tertiary elevations. By 112 days after surgery fissures had become widespread and showed areas of cracking where the surface had been fractured (Fig. 4).

Regions of disruption, common on cartilage blocks from joints after 14 days, appeared either as roughening of the surfaces (Fig. 5) or as small surface pits which were particularly prevalent on the joint surfaces of animals maintained for the longer periods after surgery.

\section{Sham-operated joints}

Areas of minimal distortion and disruption were evident on the majority of specimens collected from

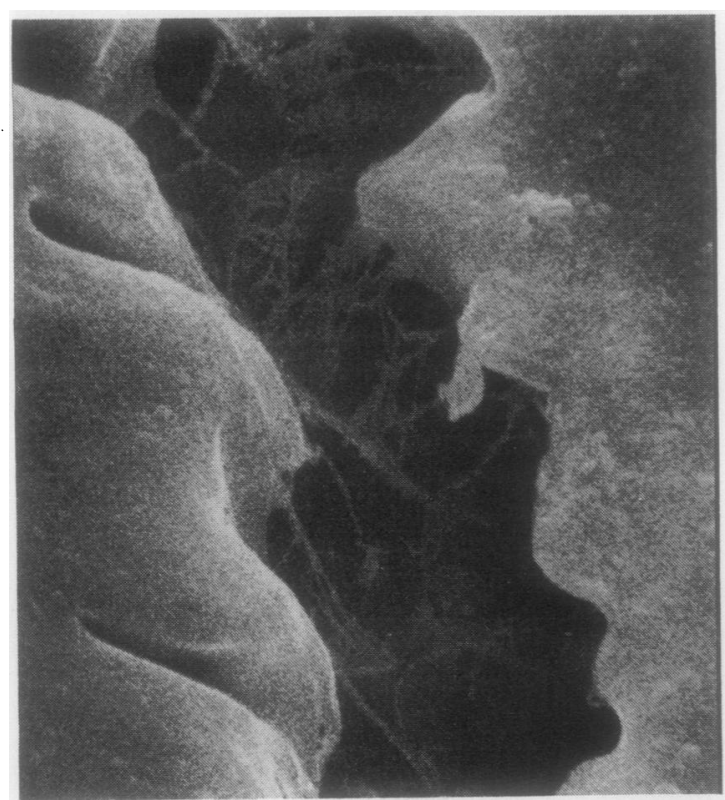

Fig. 3 Fold at the surface of medial femoral condyle 56 days after surgery. Note leasing of fibres at base of trough. LTSEM. $(\times 2010)$.

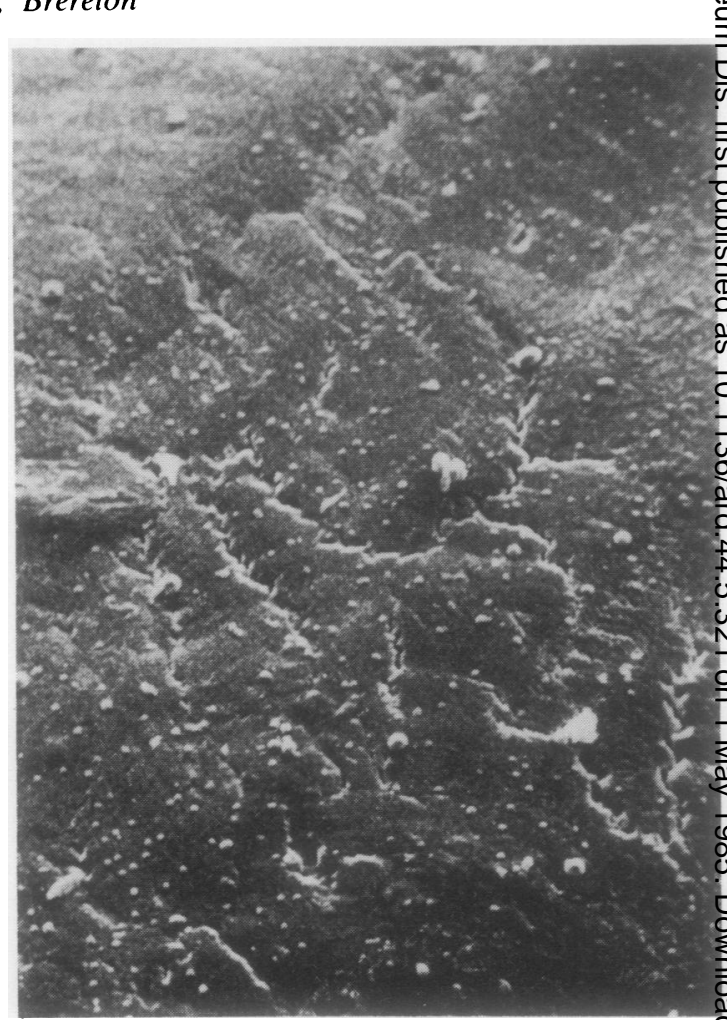

Fig. + Cracks at the articular surface 168 days after anterior cruciate ligament division. LTSEM. $(\times 110)$.

sham-operated joints 28 days or longer afte居 surgery. These regions of damage were much more. localised on tissue from sham-operated knees tharw on blocks from operated joints studied at simila? time intervals.

CONVENTIONAI. SEM

Normal joints

Articular surfaces were covered by arrays of tertiar? undulations of three types: empty hollows, elevas tions, or most frequently, elevations circumscribe by moats (Fig. 6). Tertiary features were often is figure-of-eight configurations (Fig. 6). Parallel ơ criss-cross quaternary ridges $0.9-1.6 \mu \mathrm{m}$ in width were present on the surfaces of some specimens. $A^{\mu}$ magnifications above 1000 the articular surface hat a granular appearance.

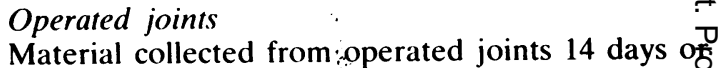
less after ACL division possessed surface feature similar to those seen on normal control tissue. Areas of normal appearance could be found in all joints examined. 


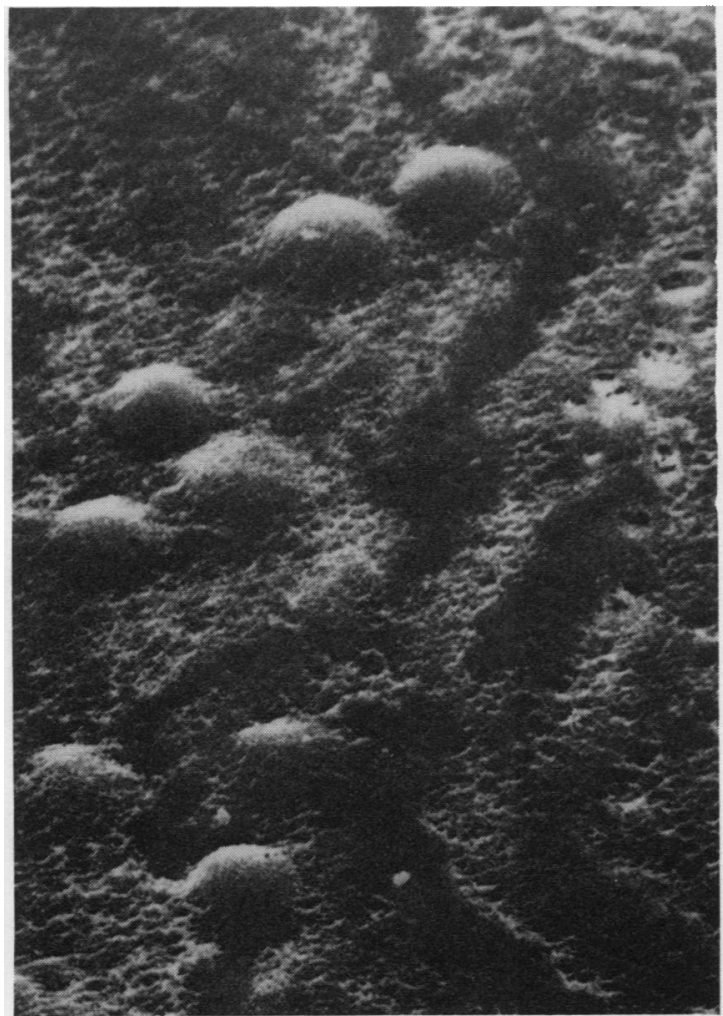

Fig. 5 Disrupted articular surface 84 days after operation. LTSEM. $(\times 1060)$.

Between one and 84 days after ACL division much of the articular surface was covered by elevations, usually circumscribed by moats, but by 112 days empty hollows were more common. Articular surfaces became more fibrous with time after surgery. At day one they were granular, by three days localised areas in which fibrils predominated could be distinguished, between 14 and 28 days the surfaces were uniformly fibrous, and by 42 days separation of the fibrils enhanced the fibrous texture. Cartilage from operated joints 56 days and later showed focal roughening of the surface, with debris and synovial fluid deposits enmeshed within frayed fibrils (Fig. 7). By 112 days holes larger in size than tertiary undulations were seen at the surface. Deformation recognised as folds accompanied roughening at 112 and 168 days. These surfaces were extremely fibrous with considerable quantities of trapped debris. In all areas of damage tertiary undulations were sparse or absent.

\section{Sham-operated joints}

Articular surfaces of blocks taken from sham- operated knees did not differ significantly from normal cartilage when examined by conventional SEM.

\section{Discussion}

Surgical division of an ACL in the dog stifle joint is an accepted animal model for inducing degenerative joint disease. The sequence of histological (Gardner, personal communication), ultrastructural, ${ }^{67}$ and biochemical ${ }^{389}$ changes that succeed operation has been reported. In the present investigation the progress of disease at the articular surface has been monitored by conventional SEM and by the recently developed method of low temperature SEM. The latter technique has been established as a method for examining fresh, unfixed, fully hydrated biological material. ${ }^{10}$

The presence of frozen deposits on the surface of some LTSEM specimens requires thermal etching to unmask the surface detail. It has been shown here

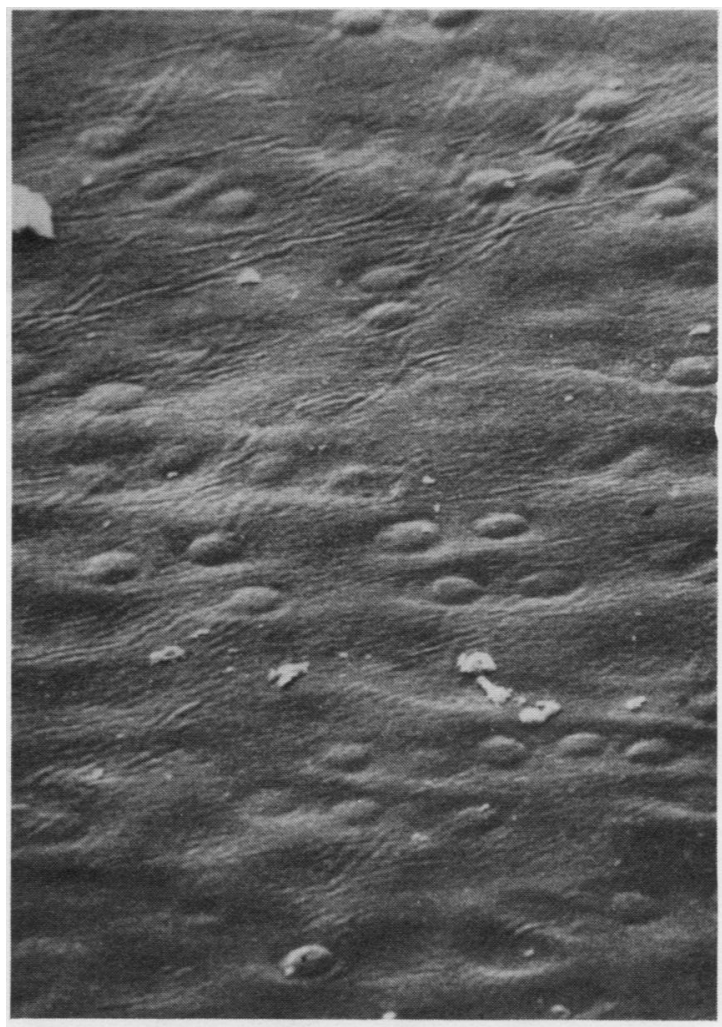

Fig. 6 Normal articular cartilage after fixation and dehydration showing tertiary features, each consisting of an elevation surrounded by a moat. Conventional SEM. $(\times 670)$. 


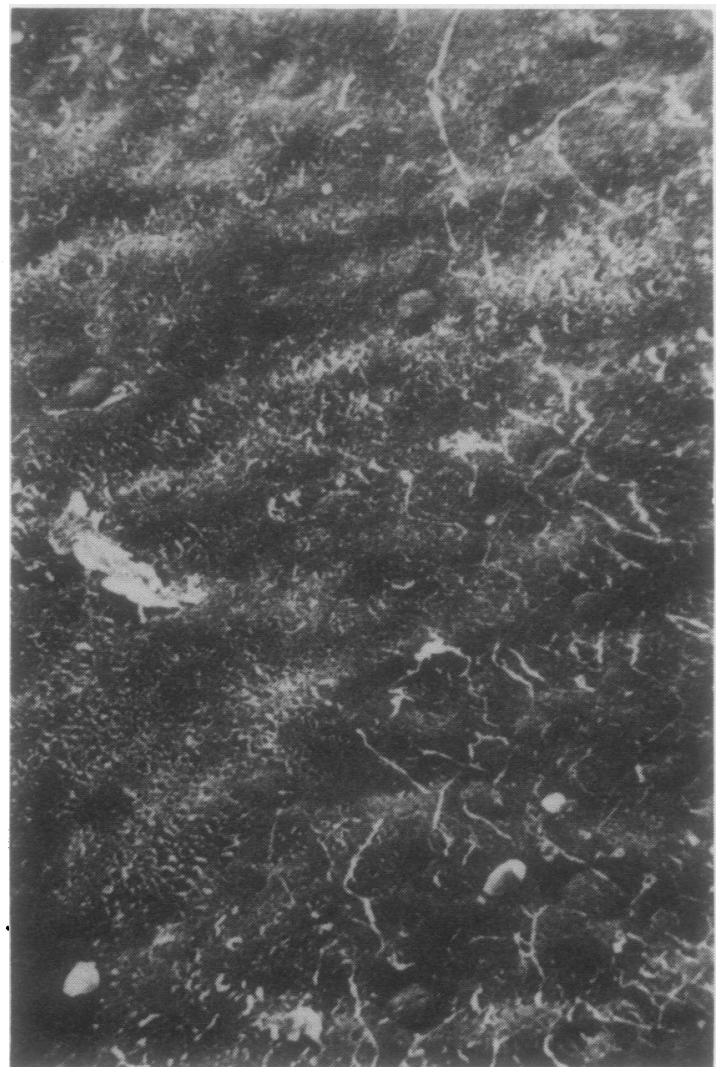

Fig. 7 Articular surface of femoral condylar cartilage 84 days after ACL division, with disrupted superficial zone and trapped debris. Conventional SEM. $(\times 620)$.

that this procedure did not alter the surface morphology. All the features described were present on both etched and non-etched material.

The en face surface structure of femoral condylar cartilage from operated joints was normal up to 14 days after surgery: the early histological and chemical changes ${ }^{89}$ were not reflected in morphological alterations at the surface. From 14 to 168 days variations in surface structure were apparent by both low temperature and conventional SEM. Changes were evident earlier than those seen by transmission electron microscopy (TEM) ${ }^{6}$ : this may be a consequence of their focal distribution. Generalised changes included a more fibrous texture and an alteration in tertiary undulations. The former may have resulted from separation of the collagen fibrils as seen by $\operatorname{TEM}^{67}$ and from a loss of proteoglycan. This feature was particularly notice- able in conventionally prepared specimens in which 맘. the shrinkage induced by the processes of fixation $\stackrel{12}{\Rightarrow}$ and dehydration would have a greater effect on the more hydrated tissue from operated joints. In frozeñ tissue elevations predominated on all cartilage blocks examined. However, in conventionally pre $\frac{\bar{\omega}}{\zeta}$ pared material there was a change from a pre $\mathbb{\$}$ ponderance of elevations surrounded by moats in normal and early postoperative material to numerous hollows in late postoperative tissue. These changes may reflect alterations in the shape and size of chondrocyte lacunae $e^{67}$ or an increase in tissue hydration ${ }^{9}$ which accompany ACL division an which are revealed by tissue shrinkage durings dehydration.

All other surface changes described were focal iriw distribution. The areas affected increased in fre quency and size with time after surgery. Folding an 9 roughening of the articular surface were observed after 14 days: such changes were accompanied by $\sqrt{7}$ loss of tertiary undulations. This may be an indica tion of cell death at the surface as seen in TEM. ${ }^{7}$ The widespread cracking in evidence 112 days aftee surgery may be synonymous with 'the flaking and peeling . . . described by Pond and $\mathrm{Nuki}^{2}$ and mays represent fibrillation. Ultimately, a severe, more generalised surface disorganisation developed which came to resemble the structural change of overt fibrillation in human osteoarthrosis. ${ }^{13}$

It is possible that damage to the cartilage surface $\overrightarrow{\overline{\hat{\rho}}}$ of the contralateral, sham-operated limbs may have been initiated by the mild sterile synovitis which wa: a result of insult to the synovial lining during division of the joint capsule. 'However, it is more probable that this damage was caused by division of the left ACL either because of an alteration in load bearing accompanying joint instability or via 2 systemic pathway, possibly mediated by somatomedin-like substance. An investigation is now underway to examine these alternatives.

The methods of LTSEM are applicable to the investigation of aspects of experimental articulan disease. It is important to reiterate the value of observations obtained in this way. Cartilage is highly hydrated, fibre-reinforced gel with largew volumes of water held within the domains of the proteoglycan molecules which are themselves associated with an intricate skeleton of type If collagen fibrils. This arrangement determines the $e^{+}$ load-bearing capacity of articular cartilage. An disorder that contributes to proteoglycan loss of degradation or to a change in water content map diminish resistance to compressive stress. In turn $\mathbb{Q}$ increased deformability leads to irreversible deg formations and finally to mechanical tissue failure 


\section{References}

1 Magnuson P B. Joint débridement. Surgical treatment of degenerative arthritis. Surg Gynecol Obstet 1941: 73: 1-9.

2 Pond M J. Nuki G. Experimentally-induced osteoarthritis in the dog. Ann Rheum Dis 1973; 32: 387-8.

3 McDevitt C A. Muir H. Pond M J. Canine articular cartilage in natural and experimentally induced osteoarthrosis. Biochem Soc Trans 1973; I: 287-9.

4 Adams M E, Billingham M E J. Animal models of degnerative joint disease. In: Berry C L, ed. Current topics in pathology. Berlin: Springer, 1982: 265-97. (Berry C L, ed. Bone and joint disease; vol. 71).

5 Gardner D L. Bradley W A, O'Connor P. Orford C R. Brereton J D. Synovitis after surgical division of the anterior cruciate ligament of the dog. Clin Exp Rheumatol 1984; 2: 11-6.

6 Orford C R, Gardner D L. O'Connor P. Ultrastructural changes in dog femoral condylar cartilage following anterior cruciate ligament section. $J$ Anat 1983; 137: 653-63.

7 Stockwell R A, Billingham M E J, Muir H. Ultrastructural changes in articular cartilage after experimental section of the anterior cruciate ligament of the dog knec. J Anat 1983: 136: 425-39.

$8 \mathrm{McDevitt}$ C A. Gilbertson E. Muir H. An experimental model of osteoarthritis; early morphological and biochemical changes. J Bone Joint Surg 1977; 59B: 24-35.

9 Carney S L. A study of the macromolecular biochemistry of articular cartilage tissue. University of Manchester. 1982. (PhD thesis.)

10 Gardner D L. O'Connor P. Oates K. Low temperature scanning electron microscopy of dog and guinea pig hyaline articular cartilage. J Anat 1981: 132: 267-82.

11 Cameron C H S, Gardner D L. Longmore R B. The preparation of human articular cartilage for scanning electron microscopy. J Microsc 1976; 108: 1-12.

12 Engfeldt B. Hjertquist S-O. Studies on the epiphyseal growth zone. 1. The preservation of glycosaminoglycans in tissues in some histotechnical procedures for electron microscopy. Virchows Arch (Cell Pathol) 1968; i: 222-9.

13 Gardner D L, Oates K, O'Connor P. Orford C R. The microscopic heterogeneity of osteoarthrosis. J Rheumatol 1983; 10 (suppl 9): 9-10. 